\title{
Semigroups and Renewal Equations on Dual Banach Spaces with Applications to Population Dynamics
}

\author{
Odo Diekmann ${ }^{1}$, Mats Gyllenberg ${ }^{2}$, and Horst R. Thieme ${ }^{3}$
}

1 Centre for Mathematics and Computer Science, P.O. Box 4079, 1009 AB Amsterdam, The Netherlands and Institute of Theoretical Biology, University of Leiden, Kaiserstraat 63, NL-2311 GP Leiden, The Netherlands

2 Department of Applied Mathematics, University of Luleå, S-951 87 Luleå, Sweden

3 Department of Mathematics, Arizona State University, Tempe, Arizona 85287, USA

\section{Introduction}

Mathematical descriptions of the dynamics of structured populations take various forms. In many cases, most notably in linear age-dependent population dynamics, integral equations form a natural modelling tool. For an age structured population in a constant environment it is possible to derive a renewal equation (Lotka's equation) for the birth rate of the population from first principles.

An other approach to structured population dynamics is to start by writing down a population balance equation. This equation takes the form of a first order hyperbolic partial differential equation describing the continuous change of individual state as well as death. The birth process is described by a boundary condition supplementing the pde.

In the case of linear age-dependent population dynamics the two approaches described above are equivalent. Integrating the population balance equation (McKendrick's equation) along characteristics one obtains the age distribution of the population in terms of the birth rate. Substituting this into the boundary condition one obtains Lotka's renewal equation. On the other hand, once Lotka's equation has been solved, one can easily write down an explicit expression for the age distribution.

The purpose of this paper is to show that the equivalence of the renewal equation and pde approaches is not confined to age structured populations but has a much wider generality. A general structured population problem can usually be formulated as an abstract Cauchy problem in $M(\Omega)$, the space of all Borel measures on the individual state space $\Omega$ (see [7]). In the linear case this Cauchy problem generates a $w^{*}$-semigroup on $M(\Omega)$ (see $\left.[2,5]\right)$. We show that a certain family of operators associated with the corresponding integrated semigroup satisfies a renewal equation on $M(\Omega)$ and conversely that the solution to this 
renewal equation uniquely determines the semigroup. All our results will be formulated in a general setting without any reference to population dynamics, but they will be illustrated by applications to age dependent population dynamics.

In section 2 we recall some facts from perturbation theory of dual semigroups as developed in [2-5] and we recall how this theory is related to Cauchy problems on dual Banach spaces. In section 3 we show how the perturbation problem gives rise to a renewal equation, the solutions of which can be used to define the solution semigroup of the problem. Finally in section 4 we take an abstract renewal equation as the starting point and we give conditions for when the solutions of this equation determine a semigroup on the dual Banach space. To achieve this goal which eventually yields the equivalence between the perturbation problem and renewal equation we introduce a new concept, that of a "multiplied integral of a semigroup". The relationship between this new notion and some more established ones like "integrated semigroup" is investigated.

\section{Perturbation theory for dual semigroups}

Let $X$ be a Banach space. Recall that a $w^{*}$-semigroup on $X^{*}$ is a family $T^{\times}=\left\{T^{\times}(t)\right\}_{t \geq 0}$ of bounded linear operators on $X^{*}$, which in addition to the semigroup properties $T^{\times}(0)=I, T^{\times}(t+s)=T^{\times}(t) T^{\times}(s)$, satisfies the continuity condition that $t \rightarrow\left\langle x, T^{\times}(t) x^{*}\right\rangle$ is continuous for any $x \in X, x^{*} \in X^{*}$. The $w^{*}$-generator $A^{\times}$of $T^{\times}$is defined by

$$
A^{\times} x^{*}=w e a k^{*}-\lim _{h \downarrow 0} \frac{1}{h}\left[T^{\times}(h) x^{*}-x^{*}\right]
$$

the domain $D\left(A^{\times}\right)$being defined as the set of all $x^{*} \in X^{*}$ for which the above limit exists. In general there is not a unique correspondence between semigroup and generator (see [6] where properties (i) and (ii) of Theorem 2.1 below are motivated).

Let $T_{0}=\left\{T_{0}(t)\right\}_{t \geq 0}$ be a strongly continuous semigroup of linear operators on $X$ with infinitesimal generator $A_{0}$. Then $T_{0}^{*}=\left\{T_{0}^{*}(t)\right\}_{t \geq 0}$ is a $w^{*}$-semigroup on $X^{*}$ with $w^{*}$-generator $A_{0}^{*}$. Define $X^{\odot}:=\overline{D\left(A_{0}^{*}\right)}$. Then $T_{0}^{\odot}$, the restriction of $T_{0}^{*}$ to $X^{\odot}$, is a strongly continuous semigroup whose generator $A_{0}^{\odot}$ is the part of $A_{0}^{*}$ in $X^{\odot}$, that is $A_{0}^{\odot} x^{\odot}=A_{0}^{*} x^{\odot}, D\left(A_{0}^{\odot}\right)=\left\{x^{\odot} \in D\left(A_{0}^{*}\right): A_{0}^{*} x^{\odot} \in X^{\odot}\right\}$.

Let $C$ be a bounded linear operator from $X^{\odot}$ into $X^{*}$. The basic perturbation result is given by the following theorem.

Theorem 2.1 Let $A^{\times}:=A_{0}^{*}+C, D\left(A^{\times}\right)=D\left(A_{0}^{*}\right)$. Then $A^{\times}$generates a $w^{*}$ semigroup $T^{\times}=\left\{T^{\times}(t)\right\}_{t \geq 0}$ on $X^{*}$ with the properties

(i) $x^{*} \in D\left(A^{\times}\right)$and $A^{\times} x^{*}=y^{*}$

if and only if

$\frac{d}{d t}\left\langle x, T^{\times}(t) x^{*}\right\rangle=\left\langle x, T^{\times}(t) y^{*}\right\rangle \forall x \in X, \forall t \geq 0$

(ii) For all $x^{*} \in X^{*}$ and all $t \geq 0$ 


$$
\int_{0}^{t} T^{\times}(\tau) x^{*} d \tau \in D\left(A^{\times}\right) \text {and } A^{\times} \int_{0}^{t} T^{\times}(\tau) x^{*} d \tau=T^{\times}(t) x^{*}-x^{*} \text {. }
$$

Note that the integral in (ii) is defined as a weak*-integral, i.e.

$$
\left\langle x, \int_{0}^{t} T^{\times}(\tau) x^{*} d \tau\right\rangle:=\int_{0}^{t}\left\langle x, T^{\times}(\tau) x^{*}\right\rangle d \tau .
$$

Theorem 2.1 can be proved in several different ways. In [6] we used a general Hille-Yosida type characterization of $w^{*}$-generators of $w^{*}$-semigroups. A perhaps more appealing approach is to first construct a strongly continuous semigroup $T^{\odot}=\left\{T^{\odot}(t)\right\}_{t \geq 0}$ on the smaller space $X^{\odot}$ by the variation of constants formula

$$
T^{\odot}(t) x^{\odot}=T_{0}^{\odot}(t) x^{\odot}+\int_{0}^{t} T_{0}^{*}(t-\tau) C T^{\odot}(\tau) x^{\odot} d \tau, x^{\odot} \in X^{\odot},
$$

and then extend $T^{\odot}$ to all of $X^{*}$. That the variation of constants formula (1) indeed defines a strongly continuous semigroup $T^{\odot}$ on $X^{\odot}$ was proved in [2]. The extension of $T^{\odot}$ to $X^{*}$ can be performed in two different ways, either through the intertwining formula

$$
T^{\times}(t):=\left(\lambda I-A^{\times}\right) T^{\odot}(t)\left(\lambda I-A^{\times}\right)^{-1},
$$

or by duality. In the latter case one obtains, after taking adjoints of $\frac{T^{\odot}(t) \text {, a }}{D(A \odot)}$ $w^{*}$-semigroup $T^{\odot *}$ on $X^{\odot *}$ and after restricting to the space $X^{\odot \odot}:=\overline{D\left(A^{\odot *}\right)}$ of strong continuity, a strongly continuous semigroup $T^{\odot \odot}$ on $X \odot \odot$. There is a duality pairing [,] between $X^{\odot \odot}$ and $X^{*}$ and hence $T^{\times}(t)$ can be defined on $X^{*}$ by

$$
\left[x^{\odot \odot}, T^{\times}(t) x^{*}\right]=\left[T^{\odot \odot}(t) x^{\odot \odot}, x^{*}\right]
$$

for all $x^{\odot \odot} \in X^{\odot \odot}$ (in particular all $x^{\odot \odot} \in X$ ), $x^{*} \in X^{*}$. The details and equivalence of these approaches are explained in [5].

Observe that a variation of constants formula like (1) is not possible for $T^{\times}$ since the perturbation $C$ is defined on $X^{\odot}$ only. (A more involved variant which also holds for $T^{\times}$on $X^{*}$ is derived in [8]). However, we will be able to write down a renewal equation for an associated family of operators on $X^{*}$ from which the semigroup $T^{\times}$can be recovered. This leads to yet another method of defining the solution semigroup on all of $X^{*}$.

We close this section by illustrating the abstract setting with an example from population dynamics.

The classical age-dependent population problem is usually formulated in the population state space $L^{1}[0, \infty)$ by the McKendrick equation supplemented by boundary and initial conditions 


$$
\begin{aligned}
& \frac{\partial n}{\partial t}+\frac{\partial n}{\partial a}=-\mu(a) n \\
& n(t, 0)=\int_{0}^{\infty} \beta(a) n(t, a) d a \\
& n(0, a)=\phi(a) .
\end{aligned}
$$

Here the age-specific fertility function $\beta$ is assumed to belong to $L^{\infty}[0, \infty)$. The problem (4) can be put in the abstract framework by considering the birth process as a perturbation of the process of aging and dying. On the predual space $X=C_{0}[0, \infty)$ the unperturbed semigroup, i.e, the solution semigroup for the problem with $\beta \equiv 0$, is given by

$$
\left[T_{0}(t) f\right](a)=e^{-\int_{a}^{a+t} \mu(\alpha) d \alpha} f(a+t) .
$$

The population state space is $X^{*}=M[0, \infty) . T_{0}^{*}$ is strongly continuous on $X^{\odot}=\overline{D\left(A_{0}^{*}\right)}$, the subspace of absolutely continuous (with respect to Lebesque measure) measures on $[0, \infty) . X^{\odot}$ can of course be identified with $L^{1}[0, \infty)$ by the Radon-Nikodym theorem, thus yielding the classical state space. The birth process is described by the bounded perturbation $C: X^{\odot} \rightarrow X^{*}$, where $C: L^{1}[0, \infty) \rightarrow M[0, \infty)$ is defined by

$$
C \phi=\langle\beta, \phi\rangle \delta=\int_{0}^{\infty} \beta(a) \phi(a) d a \delta,
$$

where $\delta$ is the Dirac measure concentrated at the origin. Thus the problem can be abstractly reformulated as the Cauchy problem

$$
\begin{aligned}
& \frac{d n}{d t}=\left(A_{0}^{*}+C\right) n, \\
& n(0)=n_{0}
\end{aligned}
$$

on $X^{*}=M[0, \infty)$. If $n_{0} \in L^{1}[0, \infty)$ then the variation of constants formula (1) yields the usual mild solution in $L^{1}[0, \infty)$.

\section{The renewal equation}

In the last section we showed how the classical McKendrick formulation of age dependent population dynamics could be viewed as an abstract perturbation problem on a dual Banach space. In order to motivate the subsequent derivation of a renewal equation associated with the perturbation problem, we start by considering the same example again.

Let the semigroup $T_{0}$ on $X=C_{0}[0, \infty)$ be given by (5) and let the perturbation $C$ be given by (6). Applying the linear functional induced by $\beta \in L^{\infty}[0, \infty)$ to both sides of the variation of constants formula (1) one obtains the equation

$$
b(t)=b_{0}(t)+\left\langle\beta, \int_{0}^{t} T_{0}^{*}(t-\tau) \delta b(\tau) d \tau\right\rangle,
$$


where

$$
b(t):=\left\langle\beta, T^{\odot}(t) x^{\odot}\right\rangle
$$

and

$$
b_{0}(t):=\left\langle\beta, T_{0}^{\odot}(t) x^{\odot}\right\rangle .
$$

The function $b(t)$ may be interpreted as the instantaneous birth rate of the population and $b_{0}(t)$ as the instantaneous birth rate of offspring of parents present in the initial population. We want to transform equation (8) into a renewal equation. To this end we note that the function $K$ defined by

$$
K(t):=\left\langle\beta, \int_{0}^{t} T_{0}^{*}(\tau) \delta d \tau\right\rangle
$$

is locally Lipschitz continuous and therefore is differentiable almost everywhere, the derivative $k$ belonging to $L_{l o c}^{\infty}[0, \infty)$ :

$$
K(t)=\int_{0}^{t} k(\tau) d \tau
$$

It is now easily seen (for details, see [3]) that $b$ satisfies the renewal equation

$$
b(t)=b_{0}(t)+\int_{0}^{t} k(t-\tau) b(\tau) d \tau .
$$

Equation (13) is nothing but Lotka's integral equation. Once $b$ is solved from (13), the solution is obtained on $X^{\odot}=L^{1}[0, \infty)$ by the explicit formula

$$
T^{\odot}(t) x^{\odot}=T_{0}^{\odot}(t) x^{\odot}+\int_{0}^{t} T_{0}^{*}(t-\tau) \delta b(\tau) d \tau .
$$

As a matter of fact we obtain the solution on all of $X^{*}=M[0, \infty)$, not in terms of the instantaneous birth rate, but in terms of the cumulative number of births. Exactly as in the case of $K$ above, we see that

$$
B_{0}(t):=\left\langle\beta, \int_{0}^{t} T_{0}^{*}(\tau) x^{*} d \tau\right\rangle
$$

defines a locally Lipschitz continuous function $B_{0}$. Therefore the renewal equation

$$
B(t)=B_{0}(t)+\int_{0}^{t} K(t-\tau) d B(\tau)
$$

has a unique solution $B$, which is again locally Lipschitz continuous. It follows that $B$ has a derivative $b \in L_{l o c}^{\infty}[0, \infty)$ almost everywhere:

$$
B(t)=\int_{0}^{t} b(\tau) d \tau
$$


If $x^{*}=x^{\odot} \in X^{\odot}$, then $b$ is of course the continuous function defined by (9). It follows that $B(t)$ is the cumulative number of births in the time interval $[0, t]$. We have the following explicit representation of the perturbed semigroup $T^{\times}$on $X^{*}$ :

$$
T^{\times}(t) x^{*}=T_{0}^{*}(t) x^{*}+\int_{0}^{t} T_{0}^{*}(t-\tau) \delta d B(\tau) .
$$

The crucial "trick" in the derivation above was integration with respect to time. We will now extend this idea to the general theory. It will therefore come as no surprise that integrated semigroups will play a key role.

If one integrates the variation of constants formula (1) from 0 to $t$ one obtains

$$
\begin{aligned}
\int_{0}^{t} T^{\odot}(\tau) x^{\odot} d \tau & =\int_{0}^{t} T_{0}^{\odot}(\tau) x^{\odot} d \tau \\
& +\int_{0}^{t} T_{0}^{*}(t-u) C \int_{0}^{u} T^{\odot}(\tau) x^{\odot} d \tau d u .
\end{aligned}
$$

Applying the operator $C: X^{\odot} \rightarrow X^{*}$ to both sides of equation (19) one obtains

$$
\begin{aligned}
C \int_{0}^{t} T^{\odot}(\tau) x^{\odot} d \tau & =C \int_{0}^{t} T_{0}^{\odot}(\tau) x^{\odot} d \tau \\
& +C \int_{0}^{t} T_{0}^{*}(t-u) C \int_{0}^{u} T^{\odot}(\tau) x^{\odot} d \tau d u .
\end{aligned}
$$

Observe that since $\int_{0}^{t} T_{0}^{*}(\tau) x^{*} d \tau \in X^{\odot}$ and $\int_{0}^{t} T^{\times}(\tau) x^{*} d \tau \in X^{\odot}$ for all $x^{*} \in X^{*}$, all terms in equations (19) and (20) still make sense if $x^{\odot} \in X^{\odot}$ is replaced by $x^{*} \in X^{*}, T_{0}^{\odot}$ by $T_{0}^{*}$ and $T^{\odot}$ by $T^{\times}$. Introducing the integrated semigroups

$$
\begin{aligned}
S_{0}^{*}(t) & =\int_{0}^{t} T_{0}^{*}(\tau) d \tau, \\
S^{\times}(t) & =\int_{0}^{t} T^{\times}(\tau) d \tau,
\end{aligned}
$$

mapping $X^{*}$ into $X^{\odot}$ and the operators

$$
\begin{aligned}
& V_{0}(t)=C S_{0}^{*}(t), \\
& V(t)=C S^{\times}(t),
\end{aligned}
$$

mapping $X^{\odot}$ into $X^{*}$ and integrating by parts we arrive at the representation

$$
S^{\times}(t)=S_{0}^{*}(t)+\int_{0}^{t} S_{0}^{*}(t-\tau) d V(\tau)
$$

of $S^{\times}(t)$, where $V(t)$ is the solution of the abstract renewal equation 


$$
V(t)=V_{0}(t)+\int_{0}^{t} V_{0}(t-\tau) d V(\tau)
$$

Once $S^{\times}(t)$ has been obtained from (26) one gets the semigroup $T^{\times}(t)$ by differentiation with respect to the weak $k^{*}$-topology:

$$
T^{\times}(t) x^{*}=\frac{d^{*}}{d t} S^{\times}(t) x^{*}=T_{0}^{*}(t) x^{*}+\int_{0}^{t} T_{0}^{*}(t-\tau) d\left[V(\tau) x^{*}\right] .
$$

The Stieltjes integrals in equations (25) and (26) are in the operator norm, whereas the Stieltjes integral in (27) must be interpreted in the weak $k^{*}$-sense.

In [8] we show how the formal manipulations above can be made rigorous. There we develop a convolution calculus on a Fréchet algebra of Lipschitz continuous operators. Resolvent theory (see [9]) then implies that the renewal equation (26) has a unique solution in this algebra and that it is given by the generation expansion

$$
V(t)=\sum_{n=0}^{\infty} V_{0}^{n \star}(t)
$$

where the terms in the series are powers with respect to the Stieltjes convolution

$$
(U \star V)(t)=\int_{0}^{t} U(t-\tau) d V(\tau)
$$

It follows that the integrated semigroup $S^{\times}(t)$ and the semigroup $T^{\times}(t)$ also have representations in terms of generation expansions:

$$
S^{\times}(t)=\sum_{n=0}^{\infty} S_{n}^{\times}(t)
$$

where

and

$$
S_{n+1}^{\times}(t)=\int_{0}^{t} S_{n}^{\times}(t-\tau) d V_{0}(\tau)
$$

$$
T^{\times}(t)=\sum_{n=0}^{\infty} T_{n}^{\times}(t)
$$

where

$$
T_{n+1}^{\times}(t) x^{*}=\int_{0}^{t} T_{n}^{\times}(t-\tau) d\left[V_{0}(\tau) x^{*}\right], x^{*} \in X^{*}
$$

Finally we note that (the integrated version of) Lotka's integral equation (16) is a special case of the abstract renewal equation (26). In age dependent population dynamics the perturbation $C$ is a rank one operator and hence so are $V_{0}(t)$ and $V(t)$. Equation (16) i simply the scalar component of (26), with $V_{0}(t)=B_{0}(t) \delta$ and $V(t)=B(t) \delta$. 


\section{Multiplied integrals of semigroups}

The main result of the last section was that if the perturbation $C: X^{\odot} \rightarrow X^{*}$ is given, then the perturbed semigroup $T^{\times}(t)$ on $X^{*}$ is obtained in terms of solutions of an associated renewal equation on $B\left(X^{*}\right)$, the space of bounded linear operators on $X^{*}$. In the McKendrick model of age dependent population dynamics it was clear how to define $C$, but this is not longer true in more general models of structured populations. If for instance the individual state space $\Omega$ is multidimensional (a subset of $R^{n}$ with $n \geq 2$ ), then there is no reasonable representation of $X^{\odot}$ as a space of functions or measures, and hence it is not clear where exactly the instantan eous birth rate operator $C$ should be defined. In some concrete models it is not even clear from biological arguments how to define $C$, see e.g. [12]. However, it is usually possible to define directly the cumulative birth function of a given population in $M(\Omega)$. In the case of multidimensional individual state space this is not a scalar, but a measure-valued function. Moreover, cumulative births, not rates, are what one can actually measure.

We are thus led to the following converse problem : Given a $w^{*}$-semigroup $T_{0}^{*}$ on $X^{*}$ and a family $\left\{V_{0}(t)\right\}_{t \geq 0}$ of locally Lipschitz continuous operators in $\mathcal{B}\left(X^{*}\right)$, under what conditions does the formula

$$
T^{\times}(t) x^{*}=T_{0}^{*}(t) x^{*}+\int_{0}^{t} T_{0}^{*}(t-\tau) d\left[V(\tau) x^{*}\right],
$$

where $V$ is the solution of the renewal equation

$$
V(t)=V_{0}(t)+\int_{0}^{t} V_{0}(t-\tau) d V(\tau)
$$

define a $w^{*}$-semigroup $T^{\times}$on $X^{*}$ ? Does there exist a bounded linear operator $C: X^{\odot} \rightarrow X^{*}$ such that $V(t)=C S^{\times}(t)$, where $S^{\times}(t)=\int_{0}^{t} T^{\times}(\tau) d \tau$ ? The rest of this section is concerned with these and related questions. We start with some definitions.

Definition 4.1 (see [6]) A $w^{*}$-semigroup $T^{\times}$on $X^{*}$ is called an integral $w^{*}$ semigroup if

$$
T^{\times}(t) \int_{0}^{s} T^{\times}(\tau) x^{*} d \tau=\int_{0}^{s} T^{\times}(t+\tau) x^{*} d \tau
$$

for all $x^{*} \in X^{*}$ and all $s, t \geq 0$.

Definition 4.2 (see $[1,10,11,13]$ ) A family $S=\{S(t)\}_{t \geq 0}$ of bounded linear operators on a Banach space is called an integrated semigroup if

$$
S(0)=0
$$

$$
t \rightarrow S(t) \text { is strongly continuous }
$$




$$
S(s) S(t)=\int_{0}^{s}[S(t+\tau)-S(\tau)] d \tau, s, t \geq 0 .
$$

It is clear from the above definitions that $T^{\times}$is an integral $w^{*}$-semigroup if and only if $S^{\times}$defined by $S^{\times}(t):=\int_{0}^{t} T^{\times}(\tau) d \tau$ is an integrated semigroup.

Definition 4.3 Let $T^{\times}$be an integral $w^{*}$-semigroup on $X^{*}$ and let $S^{\times}$be the corresponding integrated semigroup. A family $V=\{V(t)\}_{t \geq 0}$ of bounded linear operators on $X^{*}$ is called a multiplied integral of $T^{\times}$if it satisfies the following conditions

$$
V(0)=0
$$

$t \rightarrow V(t)$ is locally Lipschitz continuous

with respect to the operator norm

$$
V(s) S^{\times}(t)=\int_{0}^{t}[V(\tau+s)-V(\tau)] d \tau, t, s \geq 0 .
$$

Remark 4.4 Formal differentiation of (42) with respect to $t$ yields

$$
V(t+s)=V(t)+V(s) T^{\times}(t) .
$$

In the context of population dynamics condition (43) has a clear biological interpretation. $V(t)$ then stands for cumulative births. Let $x^{*}$ be the initial population state. In the time interval $[0, t]$ there are $V(t) x^{*}$ births while the population itself evolves to $T^{\times}(t) x^{*}$. In the time interval $[t, t+s]$ there are therefore $V(s) T^{\times}(t) x^{*}$ births. This should equal $V(t+s) x^{*}$, that is, equation (43) should hold.

Note that (42) is equivalent to

$$
V(s) S^{\times}(t)=\int_{0}^{s}[V(t+\tau)-V(\tau)] d \tau,
$$

which shows that the integrated semigroup corresponding to an integral $w^{*}$ semigroup is also a multiplied integral of that semigroup.

The terminology "multiplied integral of $T^{\times}$" introduced in Definition 4.3 is justified by the following proposition.

Proposition 4.5 Let $T^{\times}$be an integral $w^{*}$-semigroup with $w^{*}$-generator $A^{\times}$on the Banach space $X^{*}$. Let $X^{\odot}=\overline{D\left(A^{\times}\right)}$and let $C$ be a bounded linear operator from $X^{\odot}$ into $X^{*}$. Then

$$
V(t)=C S^{\times}(t), t \geq 0
$$


defines a multiplied integral of $T^{\times}$. Conversely, if $V$ is a multiplied integral of $T^{\times}$, then there exists a unique bounded linear operator $C: X^{\odot} \rightarrow X^{*}$ such that (44) holds. One has

$$
C x^{\odot}=\lim _{t \downarrow 0} \frac{1}{t} V(t) x^{\odot}
$$

for all $x^{\odot} \in X^{\odot}$.

Proof. Let $V$ be defined by (44). Then it is obvious that $V$ satisfies (40) and (41). Also,

$$
\begin{aligned}
\int_{0}^{t}[V(\tau+s)-V(\tau)] d \tau & =\int_{0}^{t} C\left[S^{\times}(\tau+s)-S^{\times}(\tau)\right] d \tau \\
& =C \int_{0}^{t}\left[S^{\times}(\tau+s)-S^{\times}(\tau)\right] d \tau \\
& =C S^{\times}(s) S^{\times}(t) \\
& =V(s) S^{\times}(t)
\end{aligned}
$$

that is, (42) holds.

Conversely, let $V$ satisfy (40) - (42). Let $x^{*} \in X^{*}, h>0$. Then

$$
\begin{aligned}
\frac{1}{h} V(h) S^{\times}(t) x^{*} & =\frac{1}{h} \int_{0}^{t}\left[V(\tau+h) x^{*}-V(\tau) x^{*}\right] d \tau \\
& \rightarrow V(t) x^{*} \text { ash } \downarrow 0 .
\end{aligned}
$$

In fact, $\frac{1}{h} V(h) S^{\times}(t) \rightarrow V(t)$ in the operator norm as $h \downarrow 0$. Since $x^{\odot}=$ $\lim _{h \downarrow 0} \int_{0}^{h} T^{\times}(\tau) \frac{1}{h} x^{\odot} d \tau, x^{\odot} \in X^{\odot}$, the set of elements of the form $S^{\times}(t) x^{*}$ is dense in $X^{\odot}$. Since moreover $\frac{1}{h}\|V(h)\| \leq L<\infty, 0<h \leq 1$, it follows that the limit in (45) exists for all $x^{\odot} \in X^{\odot}$ and defines a bounded linear operator $C: X^{\odot} \rightarrow X^{*}$. It follows from (46) that (44) holds. Uniqueness is clear.

Remark 4.6 The limit in (45) agrees with the notion of instantaneous birth rate as the derivative of cumulative number of births.

We now turn our attention to the main question of this section. Let $T_{0}=$ $\left\{T_{0}(t)\right\}_{t \geq 0}$ be a strongly continuous semigroup on $X$ and let $V_{0}$ be a multiplied integral of $T_{0}^{*}$. Let $S_{0}^{*}(t):=\int_{0}^{t} T_{0}^{*}(\tau) d \tau, t \geq 0$. Let $V(t)$ be the unique solution of the renewal equation

$$
V(t)=V_{0}(t)+\int_{0}^{t} V_{0}(t-\tau) d V(\tau)
$$

or equivalently,

$$
V(t)=V_{0}(t)+\int_{0}^{t} V(t-\tau) d V_{0}(\tau)
$$


and let $S^{\times}(t)$ be defined by

$$
S^{\times}(t)=S_{0}^{*}(t)+\int_{0}^{t} S_{0}^{*}(t-\tau) d V(\tau)
$$

Then $S^{\times}(t)$ is the unique solution of the equation

$$
S^{\times}(t)=S_{0}^{*}(t)+\int_{0}^{t} S^{\times}(t-\tau) d V_{0}(\tau)
$$

(for details, see [8]). It follows from (50) that the mapping $t \rightarrow S^{\times}(t) x^{*}$ is differentiable in the $w e a k^{*}$-sense. So we can define

$$
T^{\times}(t) x^{*}:=\frac{d^{*}}{d t} S^{\times}(t) x^{*}=T_{0}^{*}(t) x^{*}+\int_{0}^{t} T_{0}^{*}(t-\tau) d\left[V(\tau) x^{*}\right]
$$

for $x^{*} \in X^{*}$ and $t \geq 0$. It is obvious that

$$
S^{\times}(t) x^{*}=\int_{0}^{t} T^{\times}(\tau) x^{*} d \tau .
$$

Proposition 4.6 Let $C: X^{\odot} \rightarrow X^{*}$ be the unique bounded linear operator satisfying $V_{0}(t)=C S_{0}^{*}(t), t \geq 0$. Then $V(t)=C S^{\times}(t), t \geq 0$.

Proof. By (47) and (49) we have

$$
\begin{aligned}
C S^{\times}(t) & =C S_{0}^{*}(t)+C \int_{0}^{t} S_{0}^{*}(t-\tau) d V(\tau) \\
& =V_{0}(t)+\int_{0}^{t} V_{0}(t-\tau) d V(\tau) \\
& =V(t) .
\end{aligned}
$$

Proposition 4.6 shows that we are exactly in the situation described in section 3 and rigorously treated in [8]. However, the point of this section consists in deriving the main result - that $V$ is a multiplied integral of the integral $w^{*}$ semigroup $T^{\times}$- without any reference to the operator $C$.

Theorem $4.7 T^{\times}$is an integral $w^{*}$-semigroup and $V$ is a multiplied integral of $T^{\times}$.

Proof. Since $T^{\times}$and $S^{\times}$are related by (52) we have to show that $S^{\times}$is an integrated semigroup. It follows then immediately that $T^{\times}$is an integral $w^{*}$ semigroup. To this end, note that it follows from (50) that 


$$
\begin{aligned}
S^{\times}(t+\tau)-S^{\times}(\tau) & =S_{0}^{*}(t+\tau)-S_{0}^{*}(\tau) \\
& +\int_{0}^{t+\tau} S^{\times}(t+\tau-\sigma) d V_{0}(\sigma) \\
& -\int_{0}^{\tau} S^{\times}(\tau-\sigma) d V_{0}(\sigma)
\end{aligned}
$$

and hence that

$$
\begin{aligned}
\int_{0}^{s} & {\left[S^{\times}(t+\tau)-S^{\times}(\tau)\right] d \tau } \\
& =\int_{0}^{s}\left[S_{0}^{*}(t+\tau)-S_{0}^{*}(\tau)\right] d \tau \\
& +\int_{0}^{s} \int_{\tau}^{\tau+t} S^{\times}(t+\tau-\sigma) d V_{0}(\sigma) d \tau \\
& +\int_{0}^{s} \int_{0}^{\tau}\left[S^{\times}(t+\tau-\sigma)-S^{\times}(\tau-\sigma)\right] d V_{0}(\sigma) d \tau
\end{aligned}
$$

Since $S_{0}^{*}$ is an integrated semigroup the first term on the right hand side of (53) is of course equal to $S_{0}^{*}(t) S_{0}^{*}(s)$. Using the fact that $V_{0}$ is a multiplied integral of $T_{0}^{*}$, one finds that the second term to the right of (53) equals

$$
\begin{aligned}
\int_{0}^{s} & \int_{0}^{t} S^{\times}(t-\sigma) d_{\sigma}\left[V_{0}(\sigma+\tau)\right] d \tau \\
& =\int_{0}^{s} \int_{0}^{t} S^{\times}(t-\sigma) d_{\sigma}\left[V_{0}(\sigma+\tau)-V_{0}(\tau)\right] d \tau \\
& =\int_{0}^{t} S^{\times}(t-\sigma) d_{\sigma}\left[\int_{0}^{s}\left\{V_{0}(\sigma+\tau)-V_{0}(\tau)\right\} d \tau\right] \\
& =\int_{0}^{t} S^{\times}(t-\sigma) d_{\sigma}\left[V_{0}(\sigma) S_{0}^{*}(S)\right]
\end{aligned}
$$

while the third term can be written as

$$
\int_{0}^{s} \int_{0}^{s-\sigma}\left[S^{\times}(\tau+t)-S^{\times}(\tau)\right] d \tau d V_{0}(\sigma) .
$$

(The subscript $\sigma$ in $d_{\sigma}$ indicates the integration variable). Defining

$$
U_{t}(s):=\int_{0}^{s}\left[S^{\times}(\tau+t)-S^{\times}(\tau)\right] d \tau
$$

it follows from (53) that

$$
\begin{aligned}
U_{t}(s) & =S_{0}^{*}(t) S_{0}^{*}(s)+\int_{0}^{t} S^{\times}(t-\sigma) d_{\sigma}\left[V_{0}(\sigma) S_{0}^{*}(s)\right] \\
& +\int_{0}^{s} U_{t}(s-\sigma) d V_{0}(\sigma)
\end{aligned}
$$


or, by (50),

$$
U_{t}(s)=S^{\times}(t) S_{0}^{*}(s)+\int_{0}^{s} U_{t}(s-\sigma) d V_{0}(\sigma)
$$

But (47) and (48) show that $V$ is the resolvent kernel of $V_{0}$ and hence $U_{t}$, as the solution of (55), has the representation

$$
\begin{aligned}
U_{t}(s) & =S^{\times}(t) S_{0}^{*}(s)+\int_{0}^{s} S^{\times}(t) S_{0}^{*}(s-\sigma) d V(\sigma) \\
& =S^{\times}(t)\left[S_{0}^{*}(s)+\int_{0}^{s} S_{0}^{*}(s-\sigma) d V(\sigma)\right] \\
& =S^{\times}(t) S^{\times}(s),
\end{aligned}
$$

which shows that $S^{\times}$is an integrated semigroup.

It remains to be shown that $V$ is a multiplied integral of $T^{\times}$. That (40) and (41) hold is obvious. The proof that $V$ and $S^{\times}$satisfy (42) is completely analogous with the proof that $S^{\times}$is an integrated semigroup. Again using the fact that $V_{0}, S_{0}^{*}$ satisfy (42) one derives an equation similar to (55) for $U_{s}(t):=$ $\int_{0}^{t}[V(\tau+s)-V(\tau)] d \tau$, after which the resolvent representation yields the desired result.

\section{Acknowledgement}

Horst R. Thieme is supported by a Heisenberg scholarship from Deutsche Forschungsgemeinschaft.

\section{References}

1. W. Arendt (1987): Vector-valued Laplace transforms and Cauchy problems. Israel J. Math. 59, 327-352

2. Ph. Clément, O. Diekmann, M. Gyllenberg, H.J.A.M. Heijmans and H.R. Thieme (1987): Perturbation theory for dual semigroups. I. The sun-reflexive case. Math. Ann. 277, 709-725

3. Ph. Clément, O. Diekmann, M. Gyllenberg, H.J.A.M. Heijmans and H.R. Thieme (1988): Perturbation theory for dual semigroups. II. Time-dependent perturbations in the sun-reflexive case. Proc. Roy. Soc. Edinburgh 109A, 145-172

4. Ph. Clément, O. Diekmann, M. Gyllenberg, H.J.A.M. Heijmans and H.R. Thieme (1989): Perturbation theory for dual semigroups. III. Nonlinear Lipschitz continuous perturbations in the sun-reflexive case. In Volterra integrodifferential equations in Banach spaces and applications, G. Da Prato and M. Iannelli (Eds.), Pitman Research Notes in Mathematics Series 190, Longman, Harlow, 67-89

5. Ph. Clément, O. Diekmann, M. Gyllenberg, H.J.A.M. Heijmans and H.R. Thieme (1989): Perturbation theory for dual semigroups. IV. The intertwining formula and the canonical pairing. In Semigroup theory and applications, Ph. Clément, S. Invernizzi, E. Mitidieri and I.I. Vrabie (Eds.), Lecture Notes in Pure and Applied Mathematics 116, p.95-116, Marcel Dekker, New York 
6. Ph. Clément, O. Diekmann, M. Gyllenberg, H.J.A.M. Heijmans and H.R. Thieme (1989): A Hille-Yosida type theorem for a class of weakly* continuous semigroups. semigroup Forum 38, 157-178

7. O. Diekmann (1987): On the mathematical synthesis of physiological and behavioural mechanisms and population dynamics. In: Mathematical topics in population biology, morphogenesis and neurosciences, E. Teramoto and M. Yamaguli (Eds.), Springer Lecture Notes in Biomath. 71, 48-52

8. O. Diekmann, M. Gyllenberg, and H.R. Thieme: Perturbation theory of dual semigroups. V. Variation of constants formulas. submitted

9. G. Gripenberg, S-O. Londen, O. Staffans (1990): Volterra Integral and Functional Equations, Cambridge University Press, Cambridge

10. H. Kellermann and M. Hieber (1989): Integrated semigroups, J. Funct. Anal. 84, $160-180$

11. F. Neubrander (1988): Integrated semigroups and their applications to the abstract Cauchy problem. Pac. J. Math. 135, 111-155

12. H.R. Thieme (1988): Well-posedness of physiologically structured population models for Daphnia magna. How biological concepts can benefit by abstract mathematical analysis. J. Math. Biol. 26, 299-317

13. H.R. Thieme (1990): 'Integrated semigroups' and integrated solutions to abstract Cauchy problems. J. Math. Anal. Appl. 152, 416-447 